\title{
Some studies on Eisenstein series and their applications
}

\author{
H. C. Vidya and B. R. Srivatsa Kumar * \\ Department of Mathematics, Manipal Institute of Technology \\ Manipal Academy of Higher Education, Manipal-576 104, India \\ e-mails: vidyaashwath@gmail.com, sri_vatsabr@yahoo.com \\ * Corresponding author
}

Received: 21 September $2018 \quad$ Revised: 12 September $2019 \quad$ Accepted: 7 October 2019

Abstract: In the present paper, we discuss some results on Eisenstein series of level 4 and 8 . Also, as an application of this, we construct certain differential equations, incomplete integrals and deduce approximation to $3 / \pi$ and convolution sums.

Keywords: Theta functions, Eisenstein series, Convolution sums.

2010 Mathematics Subject Classification: 11M36, 14K25.

\section{Introduction}

For $q=\exp (2 \pi i \tau)$, the Dedekind $\eta$-function $\eta(\tau)$ is defined for $\operatorname{Im}(\tau)>0$ by

$$
\eta(\tau)=q^{1 / 24} \prod_{n=1}^{\infty}\left(1-q^{n}\right)
$$

where here and throughout this paper, we assume $|q|<1$. As customary, we define

$$
(a ; q)_{0}:=1, \quad(a ; q)_{n}:=\prod_{k=0}^{n-1}\left(1-a q^{k}\right) \quad \text { and } \quad(a ; q)_{\infty}:=\prod_{n=0}^{\infty}\left(1-a q^{n}\right) .
$$

For $|a b|<1$, Ramanujan's general theta-function and the Jacobi's triple product identity [9, p.35] is given by

$$
f(a, b):=\sum_{n=-\infty}^{\infty} a^{n(n+1) / 2} b^{n(n-1) / 2}=(-a ; a b)_{\infty}(-b ; a b)_{\infty}(a b ; a b)_{\infty} .
$$


The three most important special cases of $f(a, b)[9$, p.36] are

$$
\begin{gathered}
\varphi(q):=f(q, q)=\sum_{n=-\infty}^{\infty} q^{n^{2}}=\left(-q ; q^{2}\right)^{2}\left(q^{2} ; q^{2}\right)_{\infty}=\frac{(-q ;-q)_{\infty}}{(q ;-q)_{\infty}} \\
\psi(q):=f\left(q, q^{3}\right)=\sum_{n=0}^{\infty} q^{n(n+1) / 2}=\frac{\left(q^{2} ; q^{2}\right)_{\infty}}{\left(q ; q^{2}\right)_{\infty}} \\
f(-q):=f\left(-q,-q^{2}\right)=\sum_{n=-\infty}^{\infty}(-1)^{n} q^{n(3 n-1) / 2}=(q ; q)_{\infty}:=q^{-1 / 24} \eta(\tau) .
\end{gathered}
$$

Also after Ramanujan, define

$$
\chi(q):=\left(-q ; q^{2}\right)_{\infty},
$$

The Bailey's ${ }_{6} \psi_{6}$ summation formula [1] is defined as

$$
\begin{aligned}
& \sum_{n=-\infty}^{\infty} \frac{\left(q a_{1}^{1 / 2},-q a_{1}^{1 / 2}, b_{1}, c, d, e ; q\right)_{n}\left(q a_{1}^{2} / b_{1} c d e\right)^{n}}{\left(a_{1}^{1 / 2},-a_{1}^{1 / 2}, a_{1} q / b_{1}, a_{1} q / c, a_{1} q / d, a_{1} q / e ; q\right)_{n}} \\
& =\frac{\left(a_{1} q, a_{1} q / b_{1} c, a_{1} q / b_{1} d, a_{1} q / b_{1} e, a_{1} q / c d, a_{1} q / c e, a_{1} q / d e, q, q / a_{1} ; q\right)_{\infty}}{\left(a_{1} q / b_{1}, a_{1} q / c, a_{1} q / d, a_{1} q / e, q / b_{1}, q / c, q / d, q / e, q a_{1}^{2} / b_{1} c d e ; d\right)_{\infty}}
\end{aligned}
$$

where $\left(a_{1}, a_{2}, \ldots, a_{n} ; q\right)_{\infty}=\left(a_{1} ; q\right)_{\infty}\left(a_{2} ; q\right)_{\infty} \ldots\left(a_{n} ; q\right)_{\infty}$. Setting $a_{1}=a b, b_{1}=a=c, d=b=e$ in the summation formula and then replacing $q$ by $q^{4}$, we obtain

$$
\sum_{n=-\infty}^{\infty}\left[\frac{a q^{4 n}}{\left(1-a q^{4 n}\right)^{2}}-\frac{b q^{4 n}}{\left(1-b q^{4 n}\right)^{2}}\right]=a f_{4}^{6} \frac{f\left(-a b,-q^{4} / a b\right) f\left(-b / a,-a q^{4} / b\right)}{f^{2}\left(-a,-q^{4} / a\right) f^{2}\left(-b,-q^{4} / b\right)}
$$

The Ramanujan type Eisenstein's series are defined as

$$
\begin{gathered}
P(q):=1-24 \sum_{m=1}^{\infty} \frac{m q^{m}}{1-q^{m}}=1-24 \sum_{m=1}^{\infty} \sigma_{1}(m) q^{m} \\
Q(q):=1+240 \sum_{m=1}^{\infty} \frac{m^{3} q^{m}}{1-q^{m}}=1-240 \sum_{m=1}^{\infty} \sigma_{3}(m) q^{m}
\end{gathered}
$$

and

$$
R(q):=1-504 \sum_{m=1}^{\infty} \frac{m^{5} q^{m}}{1-q^{m}}=1-504 \sum_{m=1}^{\infty} \sigma_{5}(m) q^{m} .
$$

If $l$ is any positive integer, then $P_{l}$ be defined by

$$
P_{l}=P\left(q^{l}\right)
$$

Ramanujan, in his lost notebook [22], recorded some of the identities involving incomplete elliptic integrals and integrals of theta functions. The function $f$ is the Dedekind $\eta$-function as defined in (1). If

$$
v(q):=\frac{q f^{3}(-q) f^{3}\left(-q^{15}\right)}{f^{3}\left(-q^{3}\right) f^{3}\left(-q^{5}\right)}
$$


then

$$
\int_{0}^{q} f(-t) f\left(-t^{3}\right) f\left(-t^{5}\right) f\left(-t^{15}\right) d t=\frac{1}{5} \int_{2 \tan ^{-1} \frac{1}{\sqrt{5}} \sqrt{\frac{1-11 v-v^{2}}{1+v-v^{2}}}}^{2 \tan ^{-1}(1 / \sqrt{5})} \frac{d \varphi}{\sqrt{1-\frac{9}{25} \sin ^{2} \varphi}} .
$$

We have never seen the identities of the above type. Furthermore, S. Raghavan and S. S. Rangachari [20] have proved all these types of integral identities recorded by Ramanujan by employing the modular forms. For example, for four identities including the above, Raghavan and Rangachari appealed to differential equations satisfied by certain coefficients of $\eta$-functions which can be found in [15]. In an effort to find Ramanujan methods and to better understand the origin of these identities, Berndt et. al. [10] have devised the proofs independent of the theory of modular forms. Particularly they have relied on the results found in his notebooks [23] and lost notebook [22]. To prove these integrals Berndt et. al. [10] have used identities for the Eisenstein series. These relations and several $\eta$-function identities are found in Ramanujan's second notebook and also from the unorganized pages of his lost note book. Using the representations of Eisenstein series, N. D. Baruah and B. C. Berndt [8] deduced several Ramanujan-type series for $1 / \pi$. Also in [13], S. Cooper and S. Cooper and D. Ye [14] deduced some interesting series for $1 / \pi$.

In this paper, we derive some relations between Eisenstein series. Using Eisenstein series relations we construct certain differential equations. Also, using the above differential equations, we deduce certain incomplete elliptic integrals. The relations between Eisenstein series are also used to obtain an approximation to $3 / \pi$. Further, as an application, we evaluate certain convolution sums for the derived identities involving Eisenstein series. Section 2 is dedicated to record some preliminary results.

\section{Preliminary results}

For convenience, we denote $f\left(-q^{n}\right)$ by $f_{n}$ for a positive integer $n$. It is easy to see that

$$
\begin{aligned}
\varphi(-q) & =\frac{f_{1}^{2}}{f_{2}}, \quad \psi(q)=\frac{f_{2}^{2}}{f_{1}}, \quad \varphi(q)=\frac{f_{2}^{5}}{f_{1}^{2} f_{4}^{2}}, \quad \psi(-q)=\frac{f_{1} f_{4}}{f_{2}}, \\
\chi(q) & =\frac{f_{2}^{2}}{f_{1} f_{4}}, \quad \chi(-q)=\frac{f_{1}}{f_{2}} \quad \text { and } \quad f(q)=\frac{f_{2}^{3}}{f_{1} f_{4}} .
\end{aligned}
$$

Now, we define some formula for $\varphi, \psi$ at different arguments in terms of $\alpha, q$ and $z:={ }_{2} F_{1}\left(\frac{1}{2}, \frac{1}{2} ; 1 ; \alpha\right)$, recorded by Ramanujan [9, pp.122-124].

$$
\begin{aligned}
\varphi(q) & :=\sqrt{z} \\
\varphi(-q) & :=\sqrt{z}(1-\alpha)^{1 / 4}, \\
\varphi\left(-q^{2}\right) & :=\sqrt{z}(1-\alpha)^{1 / 8}, \\
\varphi\left(q^{2}\right) & :=\sqrt{\frac{z}{2}}(1+\sqrt{1-\alpha})^{1 / 2}
\end{aligned}
$$




$$
\begin{aligned}
\psi(q) & :=\sqrt{\frac{z}{2}}\left(\alpha q^{-1}\right)^{1 / 8} \\
\psi(-q) & :=\sqrt{\frac{z}{2}}\left(\alpha(1-\alpha) q^{-1}\right)^{1 / 8}
\end{aligned}
$$

Lemma 2.1. We have

$$
\begin{aligned}
\varphi(-q) \varphi(q) & =\varphi^{2}\left(-q^{2}\right), \\
\varphi(q) \psi\left(q^{2}\right) & =\psi^{2}(q) \\
\varphi^{2}(q)+\varphi^{2}(-q) & =2 \varphi^{2}\left(q^{2}\right) .
\end{aligned}
$$

Proof. The identities (13)-(15) are due to Ramanujan [21] and for a proof see [9].

Lemma 2.2. We have

$$
\begin{aligned}
& 1-x_{n}=x_{1 / n} \quad \text { and } \quad z_{1 / n}=\sqrt{n} z_{n}, \\
& P\left(e^{-2 \pi \sqrt{n}}\right)=\left(1-2 x_{n}\right) \sum_{k=0}^{\infty}(3 k+1) A_{k} X_{n}^{k}, \\
& P\left(e^{-2 \pi \sqrt{2}}\right)+P\left(e^{-\sqrt{2} \pi}\right)=\frac{6 \sqrt{2}}{\pi},
\end{aligned}
$$

where $X_{n}=4 x_{n}\left(1-x_{n}\right)$ and $A_{k}=\frac{(1 / 2)_{k}^{3}}{k !^{3}}, \quad k \geq 0$.

Proof. For a proof one can see [7,8].

Lemma 2.3. We have the following identities:

$$
\begin{aligned}
& P_{1}-2 P_{2}=\varphi^{4}(-q)-2 \varphi^{4}(q) \\
& P_{1}-4 P_{4}=-3 \varphi^{4}(q), \\
& P_{2}-2 P_{4}=8 q \psi^{4}\left(q^{2}\right)-\varphi^{4}(q) .
\end{aligned}
$$

Proof. For a proof of (19) and (20) one can see [25]. Proof of (21) follows directly by eliminating $P_{1}$ between (19) and (20).

\section{Relations between the Eisenstein series $P(q)$ and $P\left(q^{n}\right)$}

Theorem 3.1. We have

$$
P_{1}-3 P_{2}+2 P_{4}=6 \frac{\varphi^{2}\left(q^{2}\right)}{\psi^{2}(-q)}\left[\varphi^{2}\left(q^{2}\right) \psi^{2}(-q)-\varphi^{2}\left(-q^{2}\right) \psi^{2}(q)\right]
$$

Proof. Setting $a=q$ and $b=-1$ in (5), we obtain

$$
\sum_{n=-\infty}^{\infty}\left[\frac{q^{4 n+1}}{\left(1-q^{4 n+1}\right)^{2}}+\frac{q^{4 n}}{\left(1+q^{4 n}\right)^{2}}\right]=q f_{4}^{6} \frac{f\left(q, q^{3}\right) f\left(1 / q, q^{5}\right)}{f^{2}\left(-q,-q^{3}\right) f^{2}\left(1, q^{4}\right)}
$$


which is equivalent to

$$
\sum_{n=0}^{\infty} \frac{q^{2 n+1}}{\left(1-q^{2 n+1}\right)^{2}}+\sum_{n=-\infty}^{\infty} \frac{q^{4 n}}{\left(1+q^{4 n}\right)^{2}}=\frac{\varphi^{4}\left(-q^{4}\right) \psi^{2}(q)}{4 \psi^{2}(-q)}
$$

Setting $a=q^{2}$ and $b=-1$ in (5), we find that

$$
\sum_{n=-\infty}^{\infty}\left[\frac{q^{4 n+2}}{\left(1-q^{4 n+2}\right)^{2}}-\frac{q^{4 n}}{\left(1+q^{4 n}\right)^{2}}\right]=q^{2} f_{4}^{6} \frac{f\left(q^{2}, q^{2}\right) f\left(1 / q^{2}, q^{6}\right)}{f^{2}\left(-q^{2},-q^{2}\right) f^{2}\left(1, q^{4}\right)} .
$$

Using the multiplicative identity [12, Entry 25, p. 40], $\varphi^{4}\left(-q^{4}\right)=\varphi^{2}\left(q^{2}\right) \varphi^{2}\left(-q^{2}\right)$ and after simplification, we obtain

$$
2 \sum_{n=0}^{\infty} \frac{q^{4 n+2}}{\left(1-q^{4 n+2}\right)^{2}}+\sum_{n=-\infty}^{\infty} \frac{q^{4 n}}{\left(1+q^{4 n}\right)^{2}}=\frac{\varphi^{4}\left(q^{2}\right)}{4} .
$$

Using (23) in (22) and the above multiplicative identity, we obtain

$$
\sum_{n=0}^{\infty} \frac{q^{2 n+1}}{\left(1-q^{2 n+1}\right)^{2}}-2 \sum_{n=0}^{\infty} \frac{q^{4 n+2}}{\left(1-q^{4 n+2}\right)^{2}}=\frac{\varphi^{4}\left(-q^{2}\right) \psi^{2}(q)}{4 \psi^{2}(-q)}-\frac{\varphi^{4}\left(q^{2}\right)}{4} .
$$

Expanding each of these summands into a well known geometric series, then interchanging the order of the summation, adding the inner geometric series and upon using the definition of $P_{n}$, we complete the proof.

Theorem 3.2. We have

$$
P_{2}-6 P_{4}+8 P_{8}=12 \frac{\psi^{2}(-q)}{\psi^{2}(q)}\left[q \psi^{4}\left(-q^{2}\right)+\frac{\varphi^{4}\left(-q^{4}\right)}{4}\right] .
$$

Proof. Set $a=-q^{2}$ and $b=-q$ in (5), we obtain

$$
\sum_{n=-\infty}^{\infty}\left[\frac{q^{4 n+1}}{\left(1+q^{4 n+1}\right)^{2}}-\frac{q^{4 n+2}}{\left(1+q^{4 n+2}\right)^{2}}\right]=-q^{2} f_{4}^{6} \frac{f\left(-q^{3},-q\right) f\left(-1 / q,-q^{5}\right)}{f^{2}\left(q^{2}, q^{2}\right) f^{2}\left(q, q^{3}\right)}
$$

which is equivalent to

$$
\sum_{n=-\infty}^{\infty} \frac{q^{4 n+1}}{\left(1+q^{4 n+1}\right)^{2}}-2 \sum_{n=0}^{\infty} \frac{q^{2 n+2}}{\left(1+q^{2 n+2}\right)^{2}}+2 \sum_{n=0}^{\infty} \frac{q^{4 n+2}}{\left(1+q^{4 n+2}\right)^{2}}=\frac{q \psi\left(-q^{2}\right) \psi^{2}(-q)}{\psi^{2}(q)} .
$$

On setting $a=-1$ and $b=-q$ in (5), we see that

$$
\sum_{n=-\infty}^{\infty}\left[\frac{q^{4 n+1}}{\left(1+q^{4 n+1}\right)^{2}}-\frac{q^{4 n}}{\left(1+q^{4 n}\right)^{2}}\right]=f_{4}^{6} \frac{f\left(-q,-q^{3}\right)}{f^{2}\left(1, q^{4}\right) f\left(q, q^{3}\right)}
$$

which is equivalent to

$$
\sum_{n=-\infty}^{\infty} \frac{q^{4 n+1}}{\left(1+q^{4 n+1}\right)^{2}}=2 \sum_{n=1}^{\infty} \frac{q^{4 n}}{\left(1+q^{4 n}\right)^{2}}-\frac{\varphi^{4}\left(-q^{4}\right) \psi^{2}(-q)}{4 \psi^{2}(q)}+\frac{1}{4} .
$$


Using (24) in the above and on simplification, we deduce that

$$
4 \sum_{n=0}^{\infty} \frac{q^{4 n}}{\left(1+q^{4 n}\right)^{2}}-2 \sum_{n=1}^{\infty} \frac{q^{2 n}}{\left(1+q^{2 n}\right)^{2}}=\frac{\psi^{2}(-q)}{\psi^{2}(q)}\left\{q \psi^{4}\left(-q^{2}\right)+\frac{\varphi^{4}\left(-q^{4}\right)}{4}\right\}-\frac{1}{4} .
$$

We expand summands into geometric series, and then interchange the order of summation, adding the inner geometric series and finally using the definition of $P_{n}$, we obtain the result.

Theorem 3.3. We have

$$
P_{1}-8 P_{8}=-12 \frac{\psi^{2}(-q)}{\psi^{2}(q)}\left[q \psi^{4}\left(-q^{2}\right)+\frac{\varphi^{4}\left(-q^{4}\right)}{4}+\frac{7}{6} \varphi^{4}(q)+\frac{1}{6} \varphi^{4}(-q)\right] .
$$

Proof. Replacing $q$ by $q^{1 / 7}$ in (5) and then setting $a=-1$ and $b=q$, we find that

$$
P_{1}-4 P_{4}=-3 \varphi^{4}(q)
$$

Using this in Theorem 3.2, we obtain

$$
3 P_{1}-2 P_{2}-16 P_{8}=-24 \frac{\psi^{2}(-q)}{\psi^{2}(q)}\left[q \psi^{4}\left(-q^{2}\right)+\frac{\varphi^{4}\left(-q^{4}\right)}{4}\right]-9 \varphi^{4}(q) .
$$

Using (19) in (25), we complete the proof.

\section{Construction of differential equations}

Theorem 4.1. If

$$
v:=q^{1 / 8} \frac{f\left(-q^{4}\right)}{f(-q)}
$$

then

$$
\frac{q}{v} \frac{d v}{d q}=\frac{\varphi^{4}(q)}{8}
$$

Proof. By the definition of theta function, we note that

$$
v=q^{1 / 8} \frac{f\left(-q^{4}\right)}{f(-q)}=q^{1 / 8} \frac{\left(q^{4} ; q^{4}\right)_{\infty}}{(q ; q)_{\infty}}
$$

Logarithmically differentiating $v$, we deduce that

$$
\frac{1}{v} \frac{d v}{d q}=\frac{1}{q} \sum_{n=1}^{\infty} \frac{n q^{n}}{1-q^{n}}-\frac{1}{q} \sum_{n=1}^{\infty} \frac{4 n q^{4 n}}{1-q^{4 n}}+\frac{1}{8 q}
$$

By the definition of Eisenstein series, we have

$$
\frac{1}{v} \frac{d v}{d q}=\frac{1}{24 q}\left[4 P_{4}-P_{1}\right]
$$

Using (20) in the above and then rearranging the terms, we arrive at the desired result. 
Theorem 4.2. If

$$
v:=q^{7 / 24} \frac{f\left(-q^{8}\right)}{f(-q)}
$$

then

$$
\frac{q}{v} \frac{d v}{d q}=-\frac{1}{2} \frac{\psi^{2}(-q)}{\psi^{2}(q)}\left[q \psi^{4}\left(-q^{2}\right)+\frac{\varphi^{4}\left(-q^{4}\right)}{4}\right]-\frac{1}{12} \varphi^{4}(-q)-\frac{7}{12} \varphi^{4}(q) .
$$

Proof. Logarithmically differentiating $v$, we find that

$$
\begin{aligned}
\frac{1}{v} \frac{d v}{d q} & =\frac{1}{q} \sum_{n=1}^{\infty} \frac{n q^{n}}{1-q^{n}}-\frac{1}{q} \sum_{n=1}^{\infty} \frac{8 n q^{8 n}}{1-q^{8 n}}+\frac{7}{24 q} \\
& =\frac{1}{24 q}\left[P_{1}-8 P_{8}\right] .
\end{aligned}
$$

Using Theorem 3.3 in the above, we complete the proof.

Theorem 4.3. If

$$
v:=q^{1 / 24} \frac{f\left(-q^{2}\right)}{f(-q)}
$$

then

$$
\frac{q}{v} \frac{d v}{d q}=\frac{\varphi^{4}(q)}{12}-\frac{\varphi^{4}(-q)}{24}
$$

Proof. By logarithmic differentiation of $v$, we deduce that

$$
\frac{1}{v} \frac{d v}{d q}=\frac{1}{q} \sum_{n=1}^{\infty} \frac{n q^{n}}{1-q^{n}}-\frac{1}{q} \sum_{n=1}^{\infty} \frac{2 n q^{2 n}}{1-q^{2 n}}+\frac{1}{24 q} .
$$

Using the definition of Eisenstein series, we obtain

$$
\frac{q}{v} \frac{d v}{d q}=\frac{2 P_{2}-P_{1}}{24} .
$$

Using (19) in the above, we get the required result.

Theorem 4.4. If

$$
v:=q^{-5 / 24} \frac{f(-q) f\left(-q^{4}\right)}{f\left(-q^{8}\right) f\left(-q^{2}\right)}
$$

then

$$
\frac{q}{v} \frac{d v}{d q}=-\frac{\psi^{2}(-q)}{12 \psi^{2}(q)}\left[q \psi^{4}\left(-q^{2}\right)+\frac{\varphi^{4}\left(-q^{4}\right)}{4}\right]-\frac{1}{12} \varphi^{4}(-q)-\frac{1}{2} \varphi^{4}(q)-\frac{2}{3} q \psi^{4}\left(q^{2}\right) .
$$

Proof. By logarithmic differentiation of $v$, we deduce

$$
\frac{1}{v} \frac{d v}{d q}=-\frac{1}{q} \sum_{n=1}^{\infty} \frac{n q^{n}}{1-q^{n}}+\frac{1}{q} \sum_{n=1}^{\infty} \frac{2 n q^{2 n}}{1-q^{2 n}}-\frac{1}{q} \sum_{n=1}^{\infty} \frac{4 n q^{4 n}}{1-q^{4 n}}+\frac{1}{q} \sum_{n=1}^{\infty} \frac{8 n q^{8 n}}{1-q^{8 n}}-\frac{5}{24 q} .
$$

By using the definition of Eisenstein series, we have

$$
\frac{1}{v} \frac{d v}{d q}=\frac{1}{24}\left(P_{1}-2 P_{2}+4 P_{4}-8 P_{8}\right) .
$$

Using Theorem 3.2 and (21), we obtain the result. 
Theorem 4.5. If

$$
v:=\frac{f^{2}(-q) f\left(-q^{4}\right)}{f^{3}\left(-q^{2}\right)}
$$

then

$$
\frac{q}{v} \frac{d v}{d q}=\frac{\varphi^{2}\left(q^{2}\right)}{4 \psi^{2}(-q)}\left[\varphi^{2}\left(q^{2}\right) \psi^{2}(-q)-\varphi^{2}\left(-q^{2}\right) \psi^{2}(q)\right] .
$$

Proof. By logarithmic differentiation of $v$ and simplification, we find that

$$
\frac{1}{v} \frac{d v}{d q}=-\frac{2}{q} \sum_{n=1}^{\infty} \frac{n q^{n}}{1-q^{n}}+\frac{3}{q} \sum_{n=1}^{\infty} \frac{2 n q^{2 n}}{1-q^{2 n}}-\frac{1}{q} \sum_{n=1}^{\infty} \frac{4 n q^{4 n}}{1-q^{4 n}} .
$$

Now by the definition of Eisenstein series, we have

$$
\frac{q}{v} \frac{d v}{d q}=\frac{1}{12}\left(P_{1}-3 P_{2}+2 P_{4}\right) .
$$

Finally, using Theorem 3.1 in the above, we readily arrive at the required result.

Theorem 4.6. If

$$
v:=q^{1 / 4} \frac{f\left(-q^{2}\right) f^{2}\left(-q^{8}\right)}{f^{3}\left(-q^{4}\right)},
$$

then

$$
\frac{q}{v} \frac{d v}{d q}=\frac{\psi^{2}(-q)}{2 \psi^{2}(q)}\left[q \psi^{4}\left(-q^{2}\right)+\frac{\varphi^{4}\left(-q^{4}\right)}{4}\right] .
$$

Proof. Logarithmically differentiating $v$, we deduce

$$
\begin{aligned}
\frac{1}{v} \frac{d v}{d q}= & -\frac{1}{q} \sum_{n=1}^{\infty} \frac{2 n q^{2 n}}{1-q^{n}}-\frac{2}{q} \sum_{n=1}^{\infty} \frac{8 n q^{8 n}}{1-q^{8 n}}+\frac{3}{q} \sum_{n=1}^{\infty} \frac{4 n q^{4 n}}{1-q^{4 n}}+\frac{1}{4 q} \\
& =-\frac{1}{12 q}\left[P_{2}-6 P_{4}+8 P_{8}\right] .
\end{aligned}
$$

Using Theorem 3.2 in the above and on simplifying, we obtain the result.

\section{Ramanujan type series for $3 / \pi$}

Theorem 5.1. We have

$$
\frac{3}{\pi}=\sum_{k=0}^{\infty}[(8-5 \sqrt{2})(3 k+1)+(1-\sqrt{2})](2 \sqrt{2}-2)^{3 k} A_{k} .
$$

where $A_{k}=\frac{(1 / 2)_{k}^{3}}{k !^{3}}, \quad k \geq 0$.

We can prove this theorem in two methods. 
Proof. First method: Using (9)-(12) in Theorem 3.1, we obtain

$$
P_{1}-3 P_{2}+2 P_{4}=-3\left(1-x_{2}\right) z_{2}^{2}
$$

From [11, Theorem 9.2], we have $x_{2}=(\sqrt{2}-1)^{2}$. Substituting in the above, we obtain

$$
P_{1}-3 P_{2}+2 P_{4}=-6(\sqrt{2}-1) z_{2}^{2} .
$$

Setting $q=e^{-\pi / \sqrt{2}}$ and employing (18) and (20) in (26), and then comparing with (17), simplifying, we obtain the desired result.

Alternate method: By using (9) - (12) in Theorem 3.2, we deduce

$$
P_{2}-6 P_{4}+8 P_{8}=12(1-x(q))^{1 / 4}\left(1-x\left(q^{2}\right)\right)^{1 / 2} z^{2}\left(q^{2}\right)\left[\frac{x\left(q^{2}\right)^{1 / 2}}{4}+\frac{1}{4}\right] .
$$

Setting $q=e^{-\pi / \sqrt{2}}$ and using (16), we find that

$$
x\left(e^{-\pi / \sqrt{2}}\right)=1-x\left(e^{-\pi \sqrt{2}}\right)=1-x_{2}, \quad z\left(e^{-\pi / \sqrt{2}}\right)=\sqrt{2} z\left(e^{-\pi \sqrt{2}}\right)=\sqrt{2} z_{2} .
$$

Substituting these in (27), we have

$$
P_{2}-6 P_{4}+8 P_{8}=12 x_{2}^{1 / 4}\left(1-x_{2}\right)^{1 / 2} z_{2}^{2}\left[\frac{x_{2}^{1 / 2}}{4}+\frac{1}{4}\right] .
$$

From [11, Theorem 9.2], we have $x_{2}=(\sqrt{2}-1)^{2}$. Substituting in the above, we obtain

$$
P_{2}-6 P_{4}+8 P_{8}=6(\sqrt{2}-1) z_{2}^{2} .
$$

On replacing $q$ to $q^{2}$ in (20), then setting $q=e^{-\pi / \sqrt{2}}$ in the resulting identity, also using (18) in (28) and further comparing with (17), then simplifying, we complete the proof.

\section{Incomplete integrals}

Theorem 6.1. If

$$
v:=v(q):=\frac{f^{2}(-q) f\left(-q^{4}\right)}{f^{3}\left(-q^{2}\right)}
$$

then

$$
\int_{0}^{q} \frac{1}{t} \varphi^{4}(-t) d t=\int_{1}^{v(q)} \frac{8 t^{7}}{t^{8}-1} d t
$$

Proof. Using (13) and (15) in Theorem 4.5, we have

$$
\frac{q}{v} \frac{d v}{d q}=\frac{\left[\varphi^{2}(q)+\varphi^{2}(-q)\right]}{8 \psi^{2}(-q)}\left[(\varphi(q)+\varphi(-q)) \psi^{2}(-q)-2 \varphi(q) \varphi(-q) \psi^{2}(q)\right] .
$$

Using (6) and simplifying, we arrive at

$$
\frac{q}{v} \frac{d v}{d q}=\frac{1}{8}\left[1-\left(\frac{1}{v}\right)^{8}\right] \varphi^{4}(-q) .
$$


Further, it follows that

$$
\frac{\varphi^{4}(-q)}{q} d q=\frac{8 v^{7}}{v^{8}-1} d v
$$

Let $v(t)$ be defined by (29). Then the limits $t=0, q$ are transformed into $v=0, v(q)$ respectively and the result directly follows.

Theorem 6.2. If

$$
v:=v(q):=q^{1 / 4} \frac{f\left(-q^{2}\right) f^{2}\left(-q^{8}\right)}{f^{3}\left(-q^{4}\right)}
$$

then

$$
\int_{0}^{q} \frac{1}{2 \sqrt{t}} \psi^{4}(-t) d t=\int_{0}^{v(q)} \frac{4 t}{4 t^{4}+1} d t .
$$

Proof. Using (6) in Theorem 4.6 and then simplifying, we arrive at

$$
\frac{1}{2 \sqrt{q}} \psi^{4}(-q) d q=\frac{4 v}{4 v^{4}+1} d v
$$

Let $v(t)$ be defined by (30). Then the limits $t=0, q$ are transformed into $v=0, v(q)$, respectively, and the result directly follows.

\section{Convolution sums}

Let $\mathbb{N}$ denote the set of natural numbers. Let $\mathbb{Z}$ denote the set of all integers. For $k, n \in \mathbb{N}$, we set

$$
\sigma_{k}(n)=\sum_{d / n} d^{k}
$$

where $d$ runs through the positive integers dividing $n$. If $n \notin \mathbb{N}$, we set $\sigma_{k}(n)=0$. We write $\sigma(n)$ for $\sigma_{1}(n)$. For $a, b \in \mathbb{N}$ with $a \leq b$, we define the convolution sum $W_{a, b}(n)$ by

$$
W_{a, b}(n):=\sum_{a l+b m=n} \sigma(l) \sigma(m) .
$$

For all $n$, the convolution $\sum_{i+k j=n} \sigma(i) \sigma(j)$ has been evaluated explicitly for $k=1,2,3,4,5,6,7,8$, 9, 12, 16, 18 and 24, by S. Alaca, A. Alaca and K. Williams. For wonderful work one can see [2-6, 18, 19, 24, 26, 27]. Also E. X. W. Xia and O. X. M. Yao [28] derive the representations for the convolution sums $\sum_{i+6 j=n} \sigma(i) \sigma(j)$ and $\sum_{i+12 j=n} \sigma(i) \sigma(j)$.

Theorem 7.1. For all $n \in \mathbb{N}$, we have

$$
\text { i) } \begin{aligned}
\sum_{2 i+4 j=n} \sigma(i) \sigma(j)= & \frac{1}{24} \sigma\left(\frac{n}{2}\right)-\frac{5}{16} n \sigma\left(\frac{n}{2}\right)+\frac{5}{24} \sigma_{3}\left(\frac{n}{2}\right)+\frac{5}{12} n \sigma_{3}\left(\frac{n}{4}\right)-\frac{1}{12} n \sigma\left(\frac{n}{4}\right) \\
& -\frac{1}{192} A(n)+\frac{1}{18} B(n)-\frac{1}{1536} C(n),
\end{aligned}
$$




$$
\text { ii) } \begin{aligned}
\sum_{2 i+8 j=n} \sigma(i) \sigma(j)= & \frac{1}{96} \sigma\left(\frac{n}{2}\right)-\frac{1}{32} \sigma\left(\frac{n}{4}\right)-\frac{5}{16} \sigma_{3}\left(\frac{n}{4}\right)+\frac{5}{8} \sigma_{3}\left(\frac{n}{8}\right)+\frac{7}{2} \sigma\left(\frac{n}{8}\right) \\
& +\frac{25}{192} \sigma_{3}\left(\frac{n}{2}\right)-\frac{7}{32} n \sigma\left(\frac{n}{2}\right)+\frac{1}{32} n \sigma\left(\frac{n}{4}\right)-\frac{1}{6} n \sigma\left(\frac{n}{8}\right)+\frac{5}{16} n \sigma\left(\frac{n}{4}\right) \\
& -\frac{1}{192}\left[-\frac{1}{192} A(n)+\frac{1}{18} B(n)-\frac{1}{1536} C(n)-3 D(n)-\frac{1}{2} E(n)\right]
\end{aligned}
$$

and

$$
\text { iii) } \begin{aligned}
\sum_{i+8 j=n} \sigma(i) \sigma(j)= & \frac{1}{24} \sigma(n)-\frac{1}{32} n \sigma(n)+\frac{1}{24} \sigma\left(\frac{n}{8}\right)-\frac{1}{4} n \sigma\left(\frac{n}{8}\right)+\frac{5}{192} \sigma_{3}(n) \\
& +\frac{5}{3} \sigma_{3}\left(\frac{n}{8}\right)-\frac{1}{64} F(n)
\end{aligned}
$$

where

$$
\begin{aligned}
& \sum_{n=1}^{\infty} A(n) q^{n}=\frac{\varphi^{2}\left(q^{2}\right)}{\psi^{2}(-q)}\left[\varphi^{2}\left(q^{2}\right) \psi^{2}(-q)-\varphi^{2}\left(-q^{2}\right) \psi^{2}(q)\right]^{2}, \\
& \frac{1}{256}+\sum_{n=1}^{\infty} B(n) q^{n}=\left[q \psi^{4}\left(-q^{2}\right)+\frac{\varphi^{4}(q)}{16}\right]^{2}, \quad 1+\sum_{n=1}^{\infty} C(n) q^{n}=\varphi^{8}(q), \\
& \frac{1}{16}+\sum_{n=1}^{\infty} D(n) q^{n}=\frac{\psi^{4}(-q)}{\psi^{4}(q)}\left[\frac{\varphi^{4}\left(-q^{4}\right)}{4}+q \psi^{4}\left(-q^{2}\right)\right]^{2}, \\
& \frac{125}{16}+\sum_{n=1}^{\infty} E(n) q^{n}=\left(-3 \frac{\psi^{2}(-q)}{\psi^{2}(q)}\left[q \psi^{4}\left(-q^{2}\right)+\frac{\varphi^{4}\left(-q^{4}\right)}{4}\right]-\frac{11}{4} \varphi^{4}(q)-\frac{1}{2} \varphi^{4}(-q)\right)^{2}, \\
& \frac{49}{144}+\sum_{n=1}^{\infty} F(n) q^{n}=\left\{\frac{\psi^{2}(-q)}{\psi^{2}(q)}\left[q \psi^{4}\left(-q^{2}\right)+\frac{\varphi^{4}\left(-q^{4}\right)}{4}\right]+\frac{7}{24} \varphi^{4}(q)+\frac{1}{24} \varphi^{4}(-q)\right\}^{2} .
\end{aligned}
$$

Proof of $i$ ). On squaring Theorem 3.1, we obtain

$$
\begin{gathered}
P^{2}(q)+9 P^{2}\left(q^{2}\right)+4 P^{2}\left(q^{4}\right)-6 P(q) P\left(q^{2}\right)+4 P(q) P\left(q^{4}\right)-12 P\left(q^{2}\right) P\left(q^{4}\right) \\
=36 \frac{\varphi^{2}\left(q^{2}\right)}{\psi^{2}(-q)}\left[\varphi^{2}\left(q^{2}\right) \psi^{2}(-q)-\varphi^{2}\left(-q^{2}\right) \psi^{2}(q)\right]^{2} .
\end{gathered}
$$

From $[16,17]$, we have

$$
P^{2}(q)=1+\sum_{n=1}^{\infty}\left(240 \sigma_{3}(n)-288 n \sigma(n)\right) q^{n} .
$$

On employing (32) and the definition of $P\left(q^{l}\right)$ in (31) and then equating the coefficients of $q^{n}$, we obtain

$$
\begin{aligned}
& 60 \sigma_{3}(n)-72 n \sigma(n)+540 \sigma_{3}\left(\frac{n}{2}\right)-324 n \sigma\left(\frac{n}{2}\right)+12 \sigma(n)+36 \sigma\left(\frac{n}{2}\right)+240 \sigma_{3}\left(\frac{n}{4}\right) \\
& -24 \sigma\left(\frac{n}{4}\right)+72 \sigma\left(\frac{n}{2}\right)-864 \sum_{i+2 j=n} \sigma(i) \sigma(j)+576 \sum_{i+4 j=n} \sigma(i) \sigma(j)-1728 \sum_{2 i+4 j=n} \sigma(i) \sigma(j) \\
& =9 A(n),
\end{aligned}
$$

where $A(n)$ is as defined earlier. On squaring the identities (19) and (20), employing (32) and then equating the coefficients of $q^{n}$ on both sides, we have

$$
\begin{aligned}
\sum_{i+2 j=n} \sigma(i) \sigma(j)= & \frac{5}{48} \sigma_{3}(n)-\frac{1}{8} n \sigma(n)+\frac{5}{12} n \sigma_{3}\left(\frac{n}{2}\right)-\frac{1}{4} n \sigma\left(\frac{n}{2}\right) \\
& +\frac{1}{24} \sigma\left(\frac{n}{2}\right)+\frac{1}{24} \sigma(n)-\frac{1}{9} B(n),
\end{aligned}
$$


and

$$
\begin{aligned}
\sum_{i+4 j=n} \sigma(i) \sigma(j)= & \frac{5}{96} \sigma_{3}(n)-\frac{1}{16} n \sigma(n)+\frac{5}{6} n \sigma_{3}\left(\frac{n}{4}\right)-\frac{1}{4} n \sigma\left(\frac{n}{4}\right) \\
& +\frac{1}{24} \sigma\left(\frac{n}{4}\right)+\frac{1}{24} \sigma(n)-\frac{1}{512} C(n),
\end{aligned}
$$

where $B(n)$ and $C(n)$ are as defined earlier. Now on employing (34) and (35) in (33), we obtain the result.

Proof of ii). On squaring Theorem 3.2, we have

$$
\begin{gathered}
P^{2}\left(q^{2}\right)+36 P^{2}\left(q^{4}\right)+64 P^{2}\left(q^{8}\right)-12 P\left(q^{2}\right) P\left(q^{4}\right)+16 P\left(q^{2}\right) P\left(q^{8}\right)-96 P\left(q^{4}\right) P\left(q^{8}\right) \\
=144 \frac{\psi^{4}(-q)}{\psi^{4}(q)}\left[q \psi^{4}\left(-q^{2}\right)+\frac{\varphi^{4}\left(-q^{4}\right)}{4}\right]^{2} .
\end{gathered}
$$

On employing (32) and the definition of $P\left(q^{l}\right)$ in the above, then comparing the coefficients of $q^{n}$ on both sides and after simplification, we obtain

$$
\begin{aligned}
& 5 \sigma_{3}\left(\frac{n}{2}\right)-2 \sigma\left(\frac{n}{2}\right)-3 n \sigma\left(\frac{n}{2}\right)+180 \sigma_{3}\left(\frac{n}{4}\right)-54 n \sigma\left(\frac{n}{4}\right)+54 \sigma\left(\frac{n}{4}\right)-40 n \sigma\left(\frac{n}{8}\right) \\
& +320 \sigma_{3}\left(\frac{n}{8}\right)+48 \sigma\left(\frac{n}{8}\right)-144 \sum_{2 i+4 j=n} \sigma(i) \sigma(j)+192 \sum_{2 i+8 j=n} \sigma(i) \sigma(j) \\
& -1152 \sum_{4 i+8 j=n} \sigma(i) \sigma(j)=3 D(n),
\end{aligned}
$$

where $D(n)$ is as defined earlier. Subtracting (20) from Theorem 3.3, we deduce

$$
P_{4}-2 P_{8}=-3 \frac{\psi^{2}(-q)}{\psi^{2}(q)}\left[q \psi^{4}\left(-q^{2}\right)+\frac{\varphi^{4}\left(-q^{4}\right)}{4}\right]-\frac{11}{4} \varphi^{4}(q)-\frac{1}{2} \varphi^{4}(-q) .
$$

Squaring the above, then employing (32), the definition of $P\left(q^{l}\right)$ and further comparing the coefficients of $q^{n}$ on both sides, we obtain

$$
\begin{aligned}
\sum_{4 i+8 j=n} \sigma(i) \sigma(j)= & \frac{5}{48} \sigma_{3}\left(\frac{n}{4}\right)-\frac{1}{32} n \sigma\left(\frac{n}{4}\right)+\frac{5}{12} \sigma_{3}\left(\frac{n}{8}\right)-\frac{1}{16} n \sigma\left(\frac{n}{8}\right) \\
& +\frac{1}{24} \sigma\left(\frac{n}{4}\right)+\frac{5}{8} \sigma\left(\frac{n}{8}\right)-\frac{E(n)}{2304},
\end{aligned}
$$

where $E(n)$ is as defined earlier. Substituting (37) and Theorem 7.1(i) in (39) and simplifying, we obtain the result.

Proof of iii). Squaring Theorem 3.3, then using (32) and the definition of $P\left(q^{l}\right)$ and finally comparing the coefficients of $q^{n}$, we obtain the result.

\section{Acknowledgements}

The second author thank SERB, DST, India for sanctioning the project [EMR/2016/001601]. The authors thank the anonymous referees for their valuable suggestions. 


\section{References}

[1] Agarwal, R. P. (2010). Resonance of Ramanujan's Mathematics (3 Volumes), New Age International Publishers.

[2] Alaca, A., Alaca, S. \& Williams, K. S. (2006). Evaluation of the convolution sums $\sum_{l+12 m=n} \sigma(l) \sigma(m)$ and $\sum_{3 l+4 m=n} \sigma(l) \sigma(m)$, Advances in Theoretical and Applied Mathematics, 1 (1), 27-48.

[3] Alaca, S. \& Williams, K. S. (2007). Evaluation of the convolution sums $\sum_{l+6 m=n} \sigma(l) \sigma(m)$ and $\sum_{2 l+3 m=n} \sigma(l) \sigma(m)$, Journal of Number Theory, $124(2), 491-510$.

[4] Alaca, A., Alaca, S. \& Williams, K. S. (2007). Evaluation of the convolution sums $\sum_{l+18 m=n} \sigma(l) \sigma(m)$ and $\sum_{2 l+9 m=n} \sigma(l) \sigma(m)$, International Mathematical Forum. Journal for Theory and Applications, 2 (1), 45-68.

[5] Alaca, A., Alaca, S. \& Williams, K. S. (2007). Evaluation of the convolution sums $\sum_{l+24 m=n} \sigma(l) \sigma(m)$ and $\sum_{3 l+8 m=n} \sigma(l) \sigma(m)$, Mathematical Journal of Okayama University, 49, 93-111.

[6] Alaca, A., Alaca, S. \& Williams, K. S. (2008). Evaluation of the convolution sums $\sum_{m<n / 16} \sigma(l) \sigma(n-16 m)$, Canadian Mathematical Bulletin, Bulletian Canadian de Mathematiques, 51 (1), 3-14.

[7] Andrews, G. E. \& Berndt, B. C. (2009). Ramanujan's lost Notebook, Part II, Springer, New York.

[8] Baruah, N. D. \& Berndt, B. C. (2010). Eisenstein series and Ramanujan-Type series for $1 / \pi$, The Ramanujan Journal, 23 (1-3), 17-33.

[9] Berndt, B. C. (1991). Ramanujan's Notebooks, Part III, Springer.

[10] Berndt, B. C. (1994). Ramanujan's Notebooks, Part IV, Springer.

[11] Berndt, B. C. (1998). Ramanujan's Notebooks, Part V, Springer.

[12] Berndt, B. C., Choi, G., Choi, Y.-S., Hahn, H., Yeap, B. P., Yee, A. J., Yesilyurt, H. \& J. Yi. (2007). Ramanujan's forty identities for the Rogers-Ramanujan function, Mem. Amer. Math. Soc. 188 (880), 1-96.

[13] Cooper, S. (2012) Sporadic sequences, modular forms and new series for $1 / \pi$, Ramanujan J., 29, 163-183.

[14] Cooper, S. \& Ye, D. (2016). Level 14 and 15 analogous of Ramanujan's elliptic functions to alternative bases, Transactions of the American Mathematical Society, 368 (11), 7883-7910. 
[15] Fricke, R. (1922). Die Elliptic Funcktionen und Ihre Anwendungen, Bd. 2. B. G. Teubner, Leipzig.

[16] Glaisher, J. W. L. (1885). On the square of the series in which the coeff's are the sum of the divisors of the exponents, Mess. Math., 14, 156-163.

[17] Glaisher, J. W. L. (1885). Mathematical Papers, Cambridge.

[18] Huard, J. G., Ou, Z. M., Spearman, B. K. \& Williams, K. S. (2002). Elementary evaluation of certain convolution sums involving divisor functions, Number Theory for the Millennium, II, Bennett, M. A., Berndt, B. C., Boston,N., H. G. Diamond, A. J. Hildebrand and W. Philipp, Eds., 229-274.

[19] Lamire, M. \& Williams, K. S. (2006). Evaluation of two convolution sums involving the sum of divisors function, Bulletin of the Australian Mathematical Society, 73 (1), 107-115.

[20] Raghavan, S. \& Rangachari, S. S. (1989). On Ramanujan's elliptic integrals and modular identities, Oxford University Press, Bombay, 119-149.

[21] Ramanujan, S. (1957). Notebooks (2 Volumes), Bombay.

[22] Ramanujan, S. (1927). Collected Papers, Cambridge University Press, Cambridge, reprinted by Chelsea, New York, 1962, reprinted by the American Mathematical Society, Providence, RI 2000.

[23] Ramanujan, S. (1988). The Lost Notebook and Other Unpublished Papers, Narosa.

[24] Royer, E. (2007). Evaluating convolution sums of the divisor function by quasimodular forms, International Journal of Number Theory, 3 (2), 231-261.

[25] Vasuki, K. R. \& Veeresha, R. G. (2016). Ramanujan's Eisenstein series of level 7 and 14, Journal of Number Theory, 159, 59-75.

[26] Williams, K. S. (2005). The convolution sum $\sum_{m<n / 9} \sigma(l) \sigma(n-9 m)$, International Journal of Number Theory, 1 (2), 193-205.

[27] Williams, K. S. (2006). The convolution sum $\sum_{m<n / 8} \sigma(l) \sigma(n-8 m)$, Pacific Journal of Mathematics, 228 (2), 387-396.

[28] Xia, E. X. W. \& Yao, O. X. M. (2012). Eisenstein series identities involving the Borweins cubic theta functions, Journal of Applied Mathematics, Article ID 181264. 